\title{
Tuberculate supernumerary teeth and its management-a rare case report
}

\begin{abstract}
There are four types of supernumerary teeth as described by Howard ${ }^{1}$ as conical, tuberculate, supplemental and odontomas. The tuberculate type of supernumerary teeth (Figure 1-7) possesses more than one cusp or tubercle on the crown of the tooth. They rarely erupt into the oral cavity and may cause impaction of the maxillary central incisors. In this paper we describe the management of two impacted supernumerary teeth along with orthodontic management of impacted central incisors in a child where all permanent teeth have erupted into the mouth except central incisors. The purpose of this article is to discuss the surgical management, various complications associated with supernumerary teeth and the importance of early diagnosis of supernumerary teeth which might cause impaction of the incisors and space loss.
\end{abstract}

Keywords: tuberculate, supernumerary, malocclusion, diastema
Volume 7 Issue 2 - 2017

\author{
Bimal Chandra kirtaniya,' Anil Singla, ${ }^{2}$ Vikas \\ Jindal ${ }^{3}$ \\ 'Department of Paediatric Dentistry, Maharja Ganga Singh \\ Dental College, India \\ ${ }^{2}$ Department of Orthodontics, Himachal Dental College, India \\ ${ }^{3}$ Department of Periodontics, Himachal Dental College, India
}

Correspondence: Bimal Chandra Kirtaniya, Department of Paediatric Dentistry, Maharaja Ganga Singh Dental College and Research Centre, Haunmangarh Road, Sri Ganga Nagar, Rajasthan, India, Tel 9816408557,

Email drbimalchandra@gmail.com

Received: January 16, 2017 | Published: April 24, 2017

\section{Introduction}

The supernumerary tooth is the most common dental anomaly found in children and adolescent but tuberculate (Figure 1-7) type of these teeth are less frequently seen. ${ }^{2}$ The tuberculate type of supernumerary teeth have got more than one cusps or tubercle on their crown. They are often paired in occurrence and commonly located on the palatal aspect of the central incisors of maxilla. It is also described as barrel shaped tooth and the crown of the tooth may be invaginated. The root formation of such tooth is often delayed (Figure 7) and rarely they erupt into the oral cavity. These teeth cause impaction of the incisors (Figure 1-4) usually the central incisors in most of the circumstances and the present case is one of the rare condition where the child has got all permanent teeth erupted into the oral cavity except those central incisors (Figure 1). There are various complications associated with the supernumerary tooth. A mesiodens usually seen in the maxilla near midline is the most commonly occurring supernumerary tooth. It may cause mid-line diastema, rotation of incisors, crowding, ectopic eruption, impaction of incisor tooth or teeth, caries or periodontal disease. It may sometimes cause root resorption of adjacent tooth or even formation of a dentigerous cyst. ${ }^{3,4}$ These supernumerary teeth may be impacted or erupted and sometimes they may erupt into the maxillary sinus or into the nasal cavity. ${ }^{4,5}$ In other circumstances, they may not produce any pathological conditions as we have described above but they have to be removed for orthodontic tooth movements or for bone grafting in the cleft lip and palate cases or to place an implant into the jaws.

The surgical removal of impacted supernumerary tooth (Figure 1-7) is a controversial issue but most authors perform operation in the mixed dentition period when half of the root formation of adjacent permanent tooth is completed. ${ }^{6}$ If the adjacent incisors were impacted due to the presence of such extra teeth, some authors recommend exposure of the impacted permanent tooth at the same time while removing the supernumerary tooth. But the others recommend a conservative approach for it and delay for a second operation. ${ }^{7}$ The decision should be made on the basis of the impacted tooth how far it is placed into the bone and how much the impacted tooth is displaced. For example, if an impacted incisor can be clinically felt while palpation can be exposed during surgical removal of the impacted supernumerary tooth ${ }^{8}$ where as if it is lying deep into the bone should be left as such. It has been found that after removal of the supernumerary tooth and exposure of the impacted incisor, within 16 months they erupt spontaneously in about $78 \%$ cases $^{9}$ and in $30 \%$ cases require orthodontic traction. When the impacted tooth is lying deep into the bone or when it is severely displaced or when there is lack of space in the dental arch or when the impacted tooth is showing complete root formation (Figure 1-7) require complex surgery and orthodontic treatment. In such cases you may have to operate the impacted tooth more than once because of loss of attachment or other reasons. In case the impacted supernumerary tooth is lying high up into the palate and producing no complications as mentioned above should be left as such but periodic evaluation for any pathologic changes should be monitored carefully. The supernumerary tooth present in the primary dentition should not be removed if it does not create any major complications.

\section{Case report}

Ansita, a 12 year old girl was complaining of missing upper front teeth (Figure 1). On clinical examination, the upper central incisors were found to be missing from the dental arch and there was considerable space loss in the incisor region. The OPG (Figure 2) and intra-oral periapical radiograph (Figure 3) showed impaction of both the central incisors along with two impacted supernumerary teeth. The condition was explained to the parents and they agreed for surgery. Before surgical procedures were carried out, routine blood and urine examinations were done the results were within normal limit. We planned to perform the operation under local anaesthesia in the department of paediatric dentistry. After adequate anaesthesia was achieved, a full thickness of labial mucoperiosteal flap was raised. We removed minimum amount of bone from above the impacted 
supernumerary teeth and they were removed with an elevator (Figure 5-7). The flap was closed with sutures after thorough irrigation and the patient was recalled after 7 days for removal of sutures and post-operative check up. The post-operative instructions included use of prophylactic antibiotic coverage, antiseptic mouth rinse and to maintain good oral hygiene. The impacted central incisors were erupted after 3 months (Figure 8) but they were crowded due to space deficiency. The orthodontic correction was initiated soon and both the central incisors were aligned well in the dental arch (Figure 1-11).

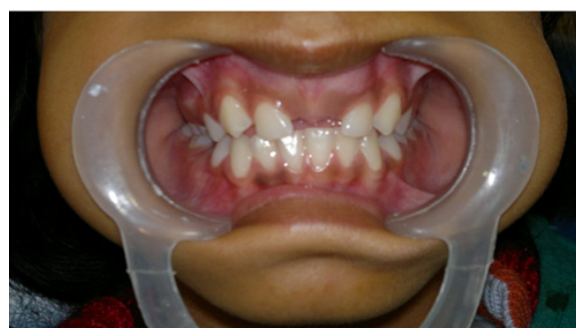

Figure I Pre-operative photograph showing impacted both the central incisors.

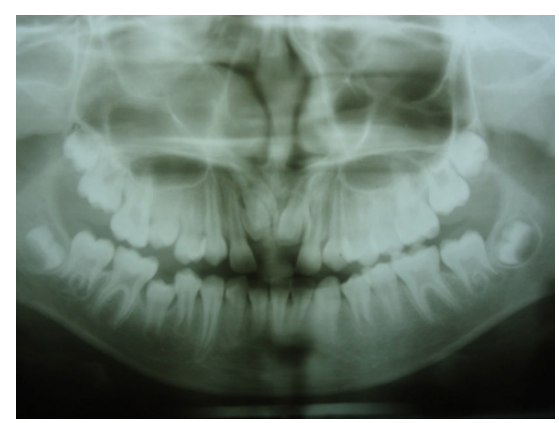

Figure 2 Pre- operative OPG showing impacted central incisors along with 2 impacted supernumerary teeth.

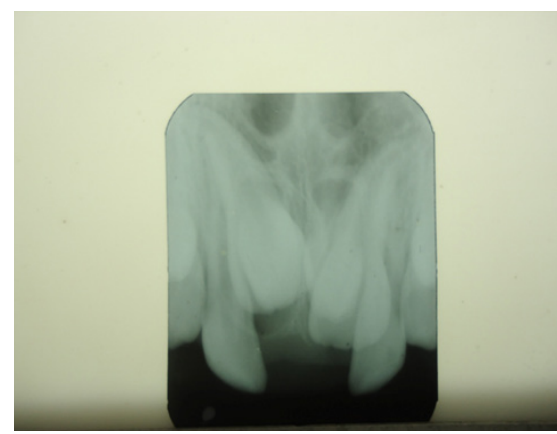

Figure 3 Pre-operative intra-oral periapical radiograph showing impacted two incisors and two impacted supernumerary teeth.

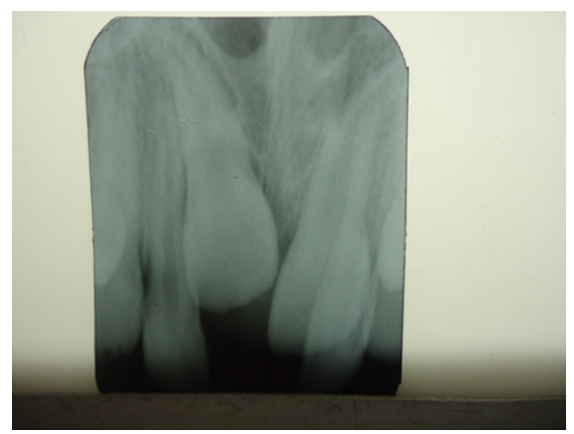

Figure 4 Post-operative radiograph showing absence of supernumerary teeth.

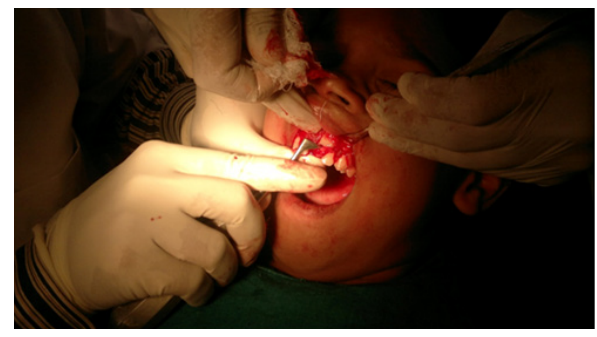

Figure 5 A full thickness of labial flap raised and an elevator is in use.

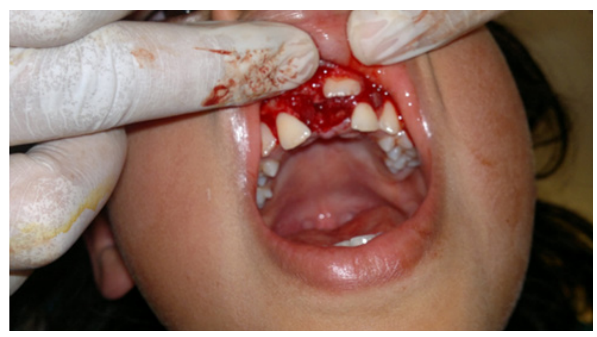

Figure 6 Both the supernumerary teeth removed.

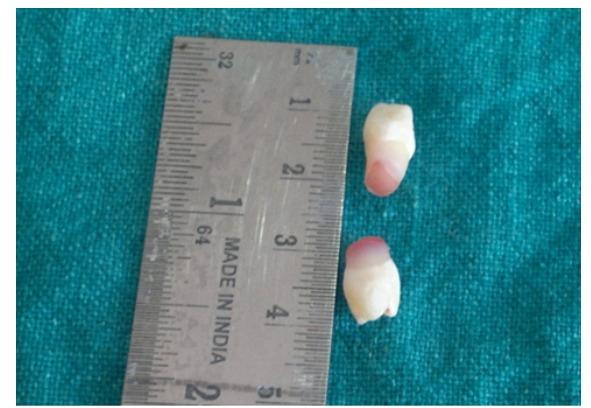

Figure 7 Extracted supernumerary teeth (note the presence cusps and under developed root).

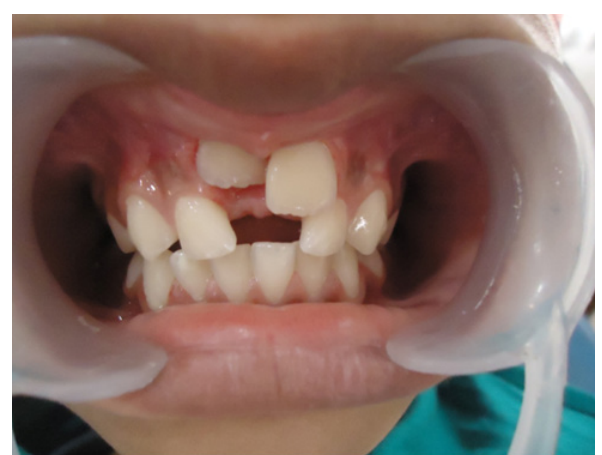

Figure 8 Erupted both central incisors but crowded.

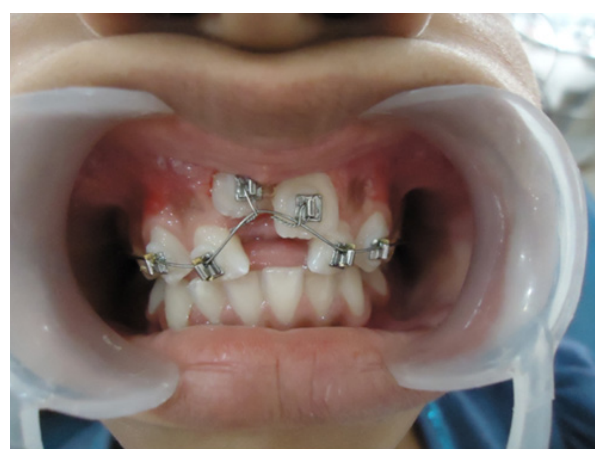

Figure 9 Orthodontic treatment started. 


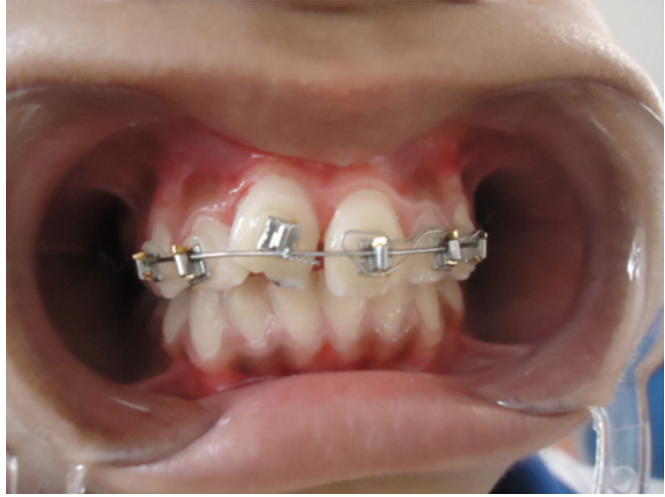

Figure 10 Mid-orthodontic treatment.

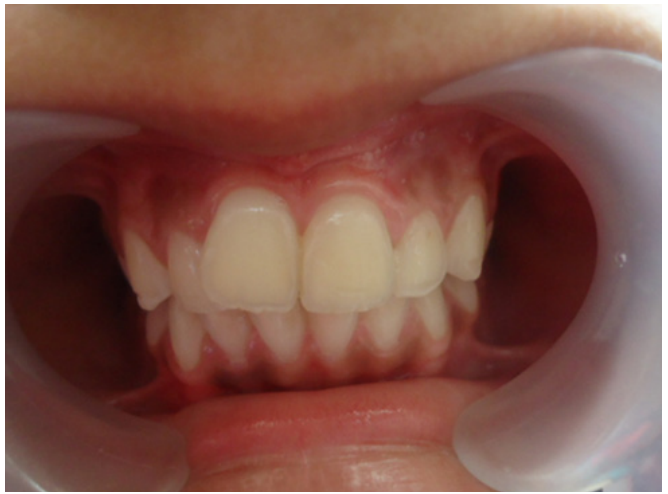

Figure I I The impacted incisors are align in the dental arch.

\section{Discussion}

A thorough clinical and radiographic evaluation of the impacted supernumerary tooth should be done before a surgical procedure is carried out. The radiographs include panoramic radiograph (Figure 2 ), intra-oral periapical radiograph (Figure $3 \& 4$ ) preferably a buccal object technique and a maxillary occlusal film. Accurate radiographs are critical for determining the position of the impacted supernumerary tooth, its relation with adjacent teeth and condition of neighbouring roots. A computed tomography (CT) is more accurate in terms of locating the supernumerary in 3 dimensions and diagnosing root resorption of the adjacent teeth but it will be costly so far Indian economy is concerned. Early diagnosis and proper interceptive intervention should be done to each child who is suspecting the presence of impacted supernumerary tooth or teeth (Figure $3 \& 4$ ) causing delayed eruption or impaction of 11 or 21 or other teeth. This will help to erupt the permanent tooth spontaneously into its place ${ }^{10}$ or to reduce the chance of development of malocclusions or dentigerous cyst formation ${ }^{3,4}$ or to prevent resorption of roots of adjacent teeth. On the other hand, if we delay the preventive and interceptive treatment to the impacted supernumerary tooth which had caused impaction of the permanent tooth (Figure $2 \& 3$ ) may cause space loss in the dental arch (Figure 1) might require complex treatment plan later on. In this present case the patient and the parents reported to us at a later stage when all the permanent teeth erupted into the oral cavity (Figure 1) except the central incisors but we could manage to align them all into the dental arch quite nicely (Figure 1-11). The aetiology of the development of supernumerary teeth is thought to be multi-factorial. The genetic influence of its development was reported by various authors but it does not follow a simple mode of the Mendelian pattern of inheritance. Usually, multiple supernumerary teeth are associated with various syndromes like Gardner syndrome, Down syndrome, cleidocranial dysplasia, cleft lip and palate which are highly correlated with heredity. Some authors recommend that hyper activity of the dental lamina is the result of various supernumerary tooth formations. They suggested that a supernumerary tooth is developed from a third tooth bud near the permanent one of the dental lamina. The other theory of its origin suggested that the tooth bud splits into two equal or unequal parts resulting in two teeth formation which are equal in size or one may be normal and the other one may be distomorphic in size and shape. This is so called the 'dichotomy theory'. This hypothesis is supported by an animal experiment in which split tooth germs have been cultivated in vitro. The supernumerary tooth seen in cleft lip and palate cases may explain this hypothesis. The supernumerary teeth in the primary dentition are rare and need not to be removed until and unless they create a problem. If they are present in the primary dentition, they may cause any one of the following consequences to the permanent dentition:

1. A normal set of permanent anterior teeth will be present.

2. A corresponding supernumerary will be present in the permanent dentition and/or

\section{A succedaneous anterior tooth will be missing.}

This observation was made by Gelling in 1984 who also reported that in $63 \%$ cases of supplemental lateral incisors in the primary dentition, there is corresponding supplemental lateral incisor in the permanent dentition. ${ }^{10}$ Nik-Hussein \& Majid ZA ${ }^{11}$ in 1996 supported this observation and found that a supernumerary tooth in the deciduous dentition is followed by an analogous supernumerary tooth in the permanent dentition and the incidence is between 35 to $60 \%{ }^{11}$ The prevalence of supernumerary teeth is said to be 1 to 3 percent in the white population in the permanent dentition. ${ }^{12}$ The prevalence of supernumerary teeth in the primary dentition is less which is 0.3 to 1.3 percent. ${ }^{13}$ In a survey of British school children in 1974 , Brook reported a prevalence of supernumerary tooth was 2.1 percent in the permanent dentition and in the primary dentition it was 0.8 percent. The author concluded that intra-uterine life is more stable environment which prevents development of supernumerary teeth in the utero hence less supernumerary teeth was found in this series of dentition. He also suggested that primary teeth were exfoliated or extracted before the children were examined may be another reason for low prevalence of the condition in the primary dentition. ${ }^{14}$ This explanation is quite reasonable unlike other authors who claimed that supernumerary in the primary dentition can accommodate in the dental arch because of spacing present in this dentition. ${ }^{13}$ Whether a supernumerary tooth is erupted or impacted in the dental arch in the permanent dentition, most often they create un-aesthetic appearance of the child which is of great concern to the parents. In a review article with 3 case reports, Kirtaniya et al. ${ }^{8}$ have shown spontaneous eruption of the impacted tooth after surgical removal of the supernumerary teeth and minor orthodontic treatment can improve the aesthetic of children considerably. ${ }^{8}$

\section{Conclusion}

Supernumerary tooth creates various types of malocclusions and other complications in the growing children. The paediatric dentist should diagnose the condition early and properly and provide preventive and interceptive treatment as early as possible. This will 
improve the aesthetic of the child and minimise the anxiety in parents as well as in children who are suffering with the condition.

\section{Acknowledgments}

None.

\section{Conflicts of interest}

The authors declare there is no conflict of interest.

\section{References}

1. Howard RD. The unerupted incisors. A study of post-operative eruptive history of incisors delayed in their eruption by supernumerary teeth. Dent Pract Dent Rec. 1967;17(9):332-341.

2. Rajendran. Shafer's textbook of oral pathology. 5th ed. Netherlands, Europe: Elsevier; 2006:1-1280.

3. Awang MN, Siar CH. Dentigerous cyst due to mesiodens: report of two cases. J Ir Dent Assoc. 1989;35(3):117-118.

4. Ray B, Bondopadhyay SN, Das D, et al. A rare cause of nasolacrimal obstruction: Dentigerous cyst in the maxillary sinus. Indian J Ophthalmol. 2009;57(6):465-467.

5. Graber TM. Orthodontic-Principle and Practice. 2001.
6. Leonard B, Kaban, Maria J Troulis. Pediatric oral and maxillofacial surgery. 2004

7. Di Biase DD. The effect of variation in tooth morphology and position on eruption. Dent Pract Dent Res. 1971;22(3):95-108.

8. Kitaniya BC, Gupta P, Sachdeva A, et al. Supernumerary teeth and their management in children. Int J Oral Health Med Res. 2016;3(2):32-37.

9. Mitchell L, Bennet TG. Supernumerary teeth causing delayed eruption, a retrospective study. Br J Orthod. 1992;19(1):41-46.

10. Gellin ME. The distribution of anomalies of primary anterior teeth and their effect on the permanent successors. Dent Clin North Am. 1984;28(1):69-80.

11. Nik Hussain NN, Majid ZA. Dental anomalies in the primary dentition: distribution and correlation with the permanent dentition. J Clin Pediatr Dent. 1996;21(1):15-19.

12. Koch H, Schwartz O, Klausen B. Indications for surgical removal of supernumerary teeth in the premaxilla. Int J Oral Maxillofac Surg. 1986;15(3):273-281.

13. Schiener MA, Sampson WJ. Supernumerary teeth: a review of the literature and four case reports. Australian dental journal. 1997;42(3):160-165.

14. Brook AH. Dental anomalies in number, form and size: their prevalence in British school children. J Int Assoc Dent Child. 1974;5(2):37-53. 